\title{
A Dynamic Optimization Model for Multi-stage Sort Plan in the Distribution Center
}

\author{
Kuo-Chung Huang, Chia-Pin Wu \\ Department of Business Administration, Nanhua University, Chiayi County, Taiwan
}

\begin{abstract}
A dynamic optimization production method is a focused approach to planning and continuous improvement for all operations and processes in the distribution center. A mathematical model is developed and presented to solve the optimal control problem in matching multi-stages production plan at any point in time. The main purpose is to investigate the optimal choice between production rate and the optimal production plan at each time through different strategies so as to achieve due date. This method provides a way for decision makers to attend to the best starting point for any given time point while operating within the best production function. Specifically in deciding is whether distribution center needs to keep operation by overtime to produce extra quantities for the next batch. Dynamic programming is also shown to be a practical method for the multi-stage optimization involved. Finally, result is generated to appraise the applicability and suitability of the proposed new multi-phase model that is solved by the developed hybrid solution approach involving a variety of uncertainties and complexities.
\end{abstract} plan.

Keywords-optimization; distribution center; production

\section{INTRODUCTION}

Flight and vehicle routing scheduling are two crucial choices to be made in strategic/ tactical and operational decision levels for global express companies. In a distribution center, the production process involving with only a few types, local ground operation team may work in advance or finish daily demand by overtime to accommodate emergency orders. These kinds of operations cost are always under variety operation human cost and fixed handling cost to accept extra orders at any time.

The hub-and-spoke distribution model is a system of connections arranged like a chariot wheel, in which all traffic moves along spokes connected to the hub at the center. The global express company adopted the hub-andspoke model for overnight package delivery; this process is referred to as make-to-order (MTO). Some practical schemes have been reported that are based on the concept of MTO. The distribution center (DC) needs to consider multiple requests when they receive shipment from different segments and a variety of demands. DC plans their production management when they receive shipment from each depot. When an order is acknowledged based on its specification (e.g., item type, quantity, next connection flight departure time), producers can create a schedule and meet production needs. Therefore, the production system aims to ensure that DC can provide service by the cut-off time in correct quantities. Then, the question involves how DC can arrange schedules in advance and prevent a negative impact when urgent extra orders are received from depots. This issue is important when production schedules are established.

Many research studies have been conducted in the field to identify resources with limited i.e. batch size, due date. The findings of such studies examining the use of various forms of resource-based methods have been discussed. Overall, there has been relatively little progress related to production speeds until recent developments. Production speed is related to production function: starting at time $t$ will ensure full operation before the due date. Production speed will affect both the profit and cost and can be treated as an important element in the overall decision-making process (Chen \& Chu, 2003). We intend to introduce the notion of production speed in production function. If the whole system is in full operation prematurely, resources may be wasted. But, if DC decides to produce too late, the said factory may not be able to meet the due date, leading to potential loss of credit and other penalties. Within capacity utilization, it is important to determine how many quantities of the next batch should be arranged in advance. This is a key point associated with optimal production systems.

Early attempts at exploring this topic have been focused on a single due date. Only Cai Fujian (2008) has proposed a framework for two stages of the production plan in an optimal control model. Therefore, this study attempts to promote this model involving the minimization of total costs via the dynamic programming approach to construct different due dates and seek optimal solutions. The purpose of this paper is to examine different due dates without capacity restriction. First, we regard batch size as a parameter, specifically in deciding whether DC needs to produce extra quantities for the next batch. Second, using a different scenario, we explore whether immediate or deferrable production would lead to optimal results related to an increase in batch size. Third, a sensitive analysis was included for each parameter.

\section{MATHEMATICAL MODELING}

The mathematical model in this paper is developed on the basis of the following assumptions:

(a) A single item with a constant rate is considered. 
(b) Production scheduling is considered only after they received an extra order. It belongs to forward scheduling, or the decision subsequent to a point in time.

(c) Demand is known.

(e) Capacity is not limited (i.e., exceeding and constant).

(f) Manufacturing costs apply to good units only.

$\mathrm{T}$.

(g) The system operates only for a prescribed period of

(h) Shortages are not allowed, input and output are balance in the distribution centers.

The following notations are used:

Parameters and given functions

\begin{tabular}{|l|l|}
\hline$[0, T]:$ & $\begin{array}{l}\text { Allowable horizon for future decision } \\
\text { making. }\end{array}$ \\
\hline$T_{n}:$ & Length of the production interval. \\
\hline$B n:$ & $\begin{array}{l}\text { Total production units during each } \\
\text { planning horizon. }\end{array}$ \\
\hline$c_{1}:$ & $\begin{array}{l}\text { The production human cost per unit time } \\
\text { with production rate. } c_{1}>0\end{array}$ \\
\hline$c_{2}:$ & $\begin{array}{l}\text { Unit handling cost per unit time. i.e. } \\
C_{2}>0 .\end{array}$ \\
\hline
\end{tabular}

\section{Decision variables and functions}

\begin{tabular}{|c|c|}
\hline$W(t):$ & $\begin{array}{l}\text { Production function at time } t, t \in[0, T] . \\
W(0)=0, W\left(T_{n}\right) \geq \sum_{n} B_{n} ; W^{\prime}(t) \text { denote } \\
\text { the production rate at } t \text {. }\end{array}$ \\
\hline$t_{w_{n}}:$ & $\begin{array}{l}\text { During } w_{n}(t), t_{w_{2}} \text { is the start point and one } \\
\text { of } \quad \text { the } \quad \text { decision } \quad \text { variables. } \\
t_{w_{n}}=\operatorname{Max}\left\{t \mid w_{n}(t)=0, t \in\left[0, T_{n}-T_{n-1}\right]\right\} \text {; } \\
w_{n}(t)=0, \quad 0 \leq t \leq t_{w_{n}} \quad \text { and } w_{n}(t)>0, \\
t_{w_{n}}<t<\left(T_{n}-T_{n-1}\right) .\end{array}$ \\
\hline$a_{n}:$ & $\begin{array}{l}\text { Total amount of extra produced units during } \\
\text { the n-th cycle } a_{n} \text { is a decision variable and } \\
W\left(T_{n}\right)=B_{n}+a_{n} ; w_{1}\left(T_{1}\right)=B_{1}+a_{1} ; a_{n} \in\left[0, B_{n-1}\right] \text {. }\end{array}$ \\
\hline
\end{tabular}

\section{MATHEMATICAL MODEL}

Time points $T_{1}, T_{2}, T_{3} \ldots$ should yield $B_{1}, B_{2}, B_{3} \ldots$, and all decision variables are known. Under infinite loading, jobs are assigned to the distribution center with regard to the capacity of the factory and are based on different due dates. Decision makers should arrange a forward scheduling plan to meet each order. At time points $T_{1}, T_{2}, T_{3} \ldots$, yield management has to decide production rates and has to operate within fixed capacities. The predicted demand is critical to the success of the yield management. For example, at $t$, operation human cost and handling cost are $c_{1}\left(W^{\prime}(t)\right)^{2}$ and $c_{2} W(t)$, respectively. The model is shown below.
Operation human cost $=\int_{T_{n-1}}^{T_{n}} C_{1}\left(W^{\prime}(t)\right)^{2} d t$; Handling cost $=\int_{T_{n-1}}^{T_{n}} c_{2}\left(W(t)-\sum_{i=0}^{n-1} B_{i}\right)$

First, let $a_{0}=0$ and $a_{n}=0$, from which one can obtain the target function,

$$
\left\{\begin{array}{l}
\operatorname{Min} \sum_{n=1}^{n} \int_{T_{n-1}}^{T_{n}}\left[c_{1}\left(W^{\prime}(t)\right)^{2}+c_{2}\left(W(t)-\sum_{i=0}^{n-1} B_{i}\right)\right] d t \\
\text { s.t. } W(0)=0, W\left(T_{n}\right) \geq \sum_{i=0}^{n} B_{n}, W^{\prime}(t) \geq 0, \forall t \in\left[0, T_{n}\right]
\end{array}\right.
$$

To find out its solution,

$$
I_{n}= \begin{cases}1,\left(B_{n}+a_{n}-a_{n-1}\right) \geq \frac{c_{2}\left(T_{n}-T_{n-1}\right)^{2}}{4 c_{1}} \\ 0,\left(B_{n}+a_{n}-a_{n-1}\right)<\frac{c_{2}\left(T_{n}-T_{n-1}\right)^{2}}{4 c_{1}}\end{cases}
$$

Then, if total production quality $\left(B_{n}+a_{n}-a_{n-1}\right)>$ $c_{2}\left(T_{n}-T_{n-1}\right)^{2} /\left(4 c_{1}\right), I_{n}=1$, the production lane should operate immediately. And, its optimal solution is shown by the following functions.

$$
w_{n}(t)=\frac{c_{2}}{4 c_{1}} t^{2}+\left[\frac{\left(B_{n}+a_{n}-a_{n-1}\right)-\frac{c_{2}}{4 c_{1}}\left(T_{n}-T_{n-1}\right)^{2}}{T_{n}-T_{n-1}}\right] t, t \in\left[0, T_{n}-T_{n-1}\right]
$$

If total production quality $\left(B_{n}+a_{n}-a_{n-1}\right)<$

$c_{2}\left(T_{n}-T_{n-1}\right)^{2} /\left(4 c_{1}\right), I_{n}=0$, the decision maker should defer to the next cycle; its function is as follows.

$$
w_{n}(t)=\frac{c_{2}}{4 c_{1}}\left(t-t_{w_{n}}\right)^{2}, t \in\left[t_{w_{n}}, T_{n}-T_{n-1}\right]
$$

\section{A DETERMINISTIC FORMULATION}

We now specify a deterministic model consistent with the above formulations. On the basis of three different due dates, we have the following cost-minimizing optimal control problem:

(I)

$$
\begin{aligned}
& \left\{\begin{array}{l}
\operatorname{Min} \sum_{n=1}^{3} \int_{T_{n-1}}^{T_{n}}\left[c_{1}\left(W^{\prime}(t)\right)^{2}+c_{2}\left(W(t)-\sum_{i=0}^{n-1} B_{i}\right)\right] d t \\
\text { s.t. } W(0)=0, W\left(T_{1}\right) \geq B_{1}, W\left(T_{2}\right) \geq B_{1}+B_{2}, W\left(T_{3}\right)=B_{1}+B_{2}+B_{3}, W^{\prime}(t) \geq 0, \forall t \in\left[0, T_{3}\right]
\end{array}\right. \\
& \text { By the method of transformation, we can simplify (I) as } \\
& \text { shown below. } \\
& \qquad \int_{0}^{T_{2}-T_{1}}\left\{c_{1}\left[W^{\prime}\left(t+T_{1}\right)\right]^{2}+c_{2}\left[W\left(t+T_{1}\right)-W\left(T_{1}\right)\right]\right\} d t+c_{2}\left[W\left(T_{1}\right)-B_{1}\right]\left(T_{2}-T_{1}\right) \\
& \quad \int_{0}^{T_{3}-T_{2}}\left\{c_{1}\left[W^{\prime}\left(t+T_{2}\right)\right]^{2}+c_{2}\left[W\left(t+T_{2}\right)-W\left(T_{2}\right)\right]\right\} d t+c_{2}\left[W\left(T_{2}\right)-B_{2}\right]\left(T_{3}-T_{2}\right)
\end{aligned}
$$

Now, under(I), we can find a feasible solution $w(t)$, where $w_{(t)}$ has a set of functions $\left(w_{1}, w_{2}, w_{3}\right)=\left(w_{1}(t), w_{2}(t), w_{3}(t)\right)$, 


$$
\begin{cases}w_{1}(t)=W(t) & , 0 \leq t \leq T_{1} \\ w_{2}(t)=W\left(t+T_{1}\right)-W\left(T_{1}\right) & , 0 \leq t \leq T_{2}-T_{1} \\ w_{3}(t)=W\left(t+T_{2}\right)-W\left(T_{2}\right) & , 0 \leq t \leq T_{3}-T_{2}\end{cases}
$$

Additionally, for any sets of functions $\left(w_{1}, w_{2}, w_{3}\right)$, $w_{1}(t)$ is in the planning horizon $\left[0, T_{1}\right]$. Its increasing function, $w_{2}(t)$, is in the planning horizon $\left[0, T_{2}-T_{1}\right]$ while $w_{3}(t)$ also is in $\left[0, T_{3}-T_{2}\right]$ increasing function. From (3.1), for $\left(w_{1}, w_{2}, w_{3}\right)$ we can find a feasible solution $W(t)$ in(I). Hence, under(I), a restriction has to be met in $W\left(T_{1}\right) \geq B_{1} \quad, \quad W\left(T_{2}\right) \geq B_{1}+B_{2}$ and $W\left(T_{3}\right)=B_{1}+B_{2}+B_{3}$. And, there should be two real numbers $a_{1}$ and $a_{2}, a_{1} \in\left[0, B_{2}\right]$ and $a_{2} \in\left[0, B_{3}\right]$. Then, $W\left(T_{1}\right)=B_{1}+a_{1}$ and $W\left(T_{2}\right)=B_{1}+B_{2}+a_{2}$, which means $w_{1}\left(T_{1}\right)=B_{1}+a_{1} \quad, \quad w_{2}\left(T_{2}-T_{1}\right)=B_{2}+a_{2}-a_{1} \quad$ 、 and $w_{3}\left(T_{3}-T_{2}\right)=B_{3}-a_{2}$. To simplify(I), the result is shown below,

$$
\text { (II) } \underset{a_{1} \in\left[0, B_{2}\right], a_{2} \in\left[0, B_{3}\right]}{\operatorname{Min}} f\left(a_{1}, a_{2}\right)
$$

Then,

(III)

$$
f\left(a_{1}, a_{2}\right)=\left\{\begin{array}{c}
\operatorname{Min}_{\left(w_{1}, w_{2}, w_{2}\right)} \int_{0}^{T_{1}}\left[c_{1}\left(w_{1}^{\prime}(t)\right)^{2}+c_{2} w_{1}(t)\right] d t+\int_{0}^{T_{2}-T_{1}}\left[c_{1}\left(w_{2}^{\prime}(t)\right)^{2}+c_{2} w_{2}(t)\right] d t \\
\quad+\int_{0}^{T_{3}-T_{2}}\left[c_{1}\left(w_{3}^{\prime}(t)\right)^{2}+c_{2} w_{3}(t)\right] d t+c_{2}\left[a_{1}\left(T_{2}-T_{1}\right)+a_{2}\left(T_{3}-T_{2}\right)\right] \\
\text { s.t. } \quad w_{1}(0)=0, w_{1}\left(T_{1}\right)=B_{1}+a_{1}, w_{1}^{\prime}(t) \geq 0, \forall t \in\left[0, T_{1}\right] \\
w_{2}(0)=0, w_{2}\left(T_{2}-T_{1}\right)=B_{2}+a_{2}-a_{1}, w_{2}^{\prime}(t) \geq 0, \forall t \in\left[0, T_{2}-T_{1}\right] \\
w_{3}(0)=0, w_{3}\left(T_{3}-T_{2}\right)=B_{3}-a_{2}, w_{3}^{\prime}(t) \geq 0, \forall t \in\left[0, T_{3}-T_{2}\right]
\end{array}\right.
$$

There are two situations: One is operated when an order is obtained(immediate production), while the other involves the distribution center deferring this order to the next cycle (deferred production). These situations arise because of the quantity of order not exceeding the level of immediate operation. Hence, in general, we consider model (III) as the general model more so than the others.

That also means $\left(w_{1}, w_{2}, w_{3}\right)$, if we define $t_{W_{1}}=\operatorname{Max}\left\{t \mid w_{1}(t)=0, t \in\left[0, T_{1}\right]\right\}$

$t_{w_{2}}=\operatorname{Max}\left\{t \mid w_{2}(t)=0, t \in\left[0, T_{2}-T_{1}\right]\right\}$ and $t_{w_{3}}=\operatorname{Max}\left\{t \mid w_{3}(t)=0, t \in\left[0, T_{3}-T_{2}\right]\right\} \quad$. Then, in model(III), $f\left(a_{1}, a_{2}\right)$ can be rewritten as follows.

$$
\begin{gathered}
\int_{t_{w_{1}}}^{T_{1}-T_{0}}\left[c_{1}\left(w_{1}^{\prime}(t)\right)^{2}+c_{2} w_{1}(t)\right] d t \quad \int_{t_{w_{2}}}^{T_{2}-T_{1}}\left[c_{1}\left(w_{2}(t)\right)^{2}+c_{2} w_{2}(t)\right] d t \\
\int_{t_{w_{3}}}^{T_{3}-T_{2}}\left[c_{1}\left(w_{3}(t)\right)^{2}+c_{2} w_{3}(t)\right] d t
\end{gathered},
$$

Hence, $f\left(a_{1}, a_{2}\right)$ is shown in model(IV)

$$
f\left(a_{1}, a_{2}\right)=\left\{\begin{aligned}
\operatorname{Min}_{w_{1}} \int_{t_{w_{1}}}^{T_{1}}\left[c_{1}\left(w_{1}^{\prime}(t)\right)^{2}+c_{2} w_{1}(t)\right] d t+\underset{w_{2}}{T_{1}} \int_{t_{w_{2}}}^{T_{2}-T_{1}}\left[c_{1}\left(w_{2}^{\prime}(t)\right)^{2}+c_{2} w_{2}(t)\right] d t \\
+\underset{w_{3}}{\operatorname{Min}} \int_{t_{3}-T_{2}}^{T_{w_{2}}}\left[c_{1}\left(w_{3}^{\prime}(t)\right)^{2}+c_{2} w_{3}(t)\right] d t+c_{2}\left[a_{1}\left(T_{2}-T_{1}\right)+a_{2}\left(T_{3}-T_{2}\right)\right] \\
\text { s.t. } \quad w_{1}\left(t_{w_{1}}\right)=0, t_{w_{1}} \geq 0, w_{1}\left(T_{1}\right)=B_{1}+a_{1}, w_{1}^{\prime}(t) \geq 0, \forall t \in\left[t_{w_{1}}, T_{1}\right] \\
\quad w_{2}\left(t_{w_{2}}\right)=0, t_{w_{2}} \geq 0, w_{2}\left(T_{2}-T_{1}\right)=B_{2}+a_{2}-a_{1}, w_{2}^{\prime}(t) \geq 0, \forall t \in\left[t_{w_{2}}, T_{2}-T_{1}\right] \\
w_{3}\left(t_{w_{3}}\right)=0, t_{w_{3}} \geq 0, w_{3}\left(T_{3}-T_{2}\right)=B_{3}-a_{2}, w_{3}^{\prime}(t) \geq 0, \forall t \in\left[t_{w_{3}}, T_{3}-T_{2}\right]
\end{aligned}\right.
$$

To give two non-negative real numbers $a_{1}$ and $a_{2}$ in (IV), we can get a feasible solution as $\left(w_{1}, w_{2}, w_{3}\right) . w_{1}(t)$, $w_{2}(t)$ and $w_{3}(t)$ would be treated as a set of functions including $a_{1}$ and $a_{2}$. Now, let $a_{1}^{*}$ and $a_{2}^{*}$ be feasible solutions for(II), $W^{*}(t)$ for (I). If we can find out a feasible solution, then they should be $a_{1}^{*}$ and $a_{2}^{*}$. This solution also results in $W^{*}(t)$. $a_{1}^{*}$ and $a_{2}^{*}$.

\section{MODEL DEVELOPMENT}

In this section, we discuss the endpoint involved for each stage, where our purpose is to minimize the average production cost for each batch. In accordance with the results (Weierstrass-Erdmann Corner Condition), we assume that each endpoint should have the same specialty as in model (I). Then, we can find out $a_{1}^{*}$ and $a_{2}^{*}$ in model(II). The evolution of the process is described by the following model (I)equation:

\section{A. Corner Condition}

While we do not consider the two restrictions $W\left(T_{1}\right) \geq B_{1}$ and $W\left(T_{2}\right) \geq B_{1}+B_{2}$ in model (I), they can be treated as a single batch for one due date. It is well known that an optimal solution certainly exists, which is assumed to meet an Euler equation when the endpoint would be determined by $W(0)=0$ and $W\left(T_{3}\right)=B_{1}+B_{2}+B_{3}$. To identify its own optimal function, we need to discuss two different cases involving immediate operation or deferral to the next cycle. These also need to satisfy $W^{\prime}(t) \geq 0$. Hence, to simplify the method needed to find an optimal solution, we assume $W^{\prime}(t) \geq 0$ and focus on both $W\left(T_{1}\right) \geq B_{1}$ and $W\left(T_{2}\right) \geq B_{1}+B_{2}$ under a single due date. In particular, there is some characteristic with $W^{*}(t)$ :

Consider the decision function $W(t)$ as an optimal function $\int_{T_{0}}^{T_{3}} F\left(t, W, W^{\prime}\right) d t$ that is subject to

$$
W\left(T_{0}\right)=W_{T_{0}}, W\left(T_{3}\right)=W_{T_{3}}
$$

Note that $W(t)$ is a Piecewise Smooth function, which means that $W(t)$ should be continuous in the individual planning horizon $\left[T_{0}, T_{3}\right]$ and be a continuous derivative that excludes certain time points. We assume $W^{*}(t)$ in (4.1) cannot be a continuous derivative at $T_{1}$ and $T_{2}$ during the planning horizon $\left[T_{0}, T_{3}\right]$.

Thus, we can find out the subjection function in (4.1) as follows 


$$
\int_{T_{0}}^{T_{3}} F\left(t, W, W^{\prime}\right) d t=\int_{T_{0}}^{T_{1}} F\left(t, W, W^{\prime}\right)+\int_{T_{1}}^{T_{2}} F\left(t, W, W^{\prime}\right) d t+\int_{T_{2}}^{T_{3}} F\left(t, W, W^{\prime}\right) d t
$$

If we suppose that $W\left(T_{1}\right)=W_{T_{1}}$ and $W\left(T_{2}\right)=W_{T_{2}}$, while $t \in\left[0, T_{1}\right]$, in model (4.1), $W^{*}(t)$ must optimize

$\int_{T_{0}}^{T_{1}} F\left(t, W, W^{\prime}\right) d t$ and is subject to

$$
W\left(T_{0}\right)=W_{T_{0}}, W\left(T_{1}\right)=W_{T_{1}}
$$

$t \in\left[T_{1}, T_{2}\right]$, in (4.1) $W^{*}(t)$ optimizes

$\int_{T_{1}}^{T_{2}} F\left(t, W, W^{\prime}\right) d t$ and is subject to

$$
W\left(T_{1}\right)=W_{T_{1}}, W\left(T_{2}\right)=W_{T_{2}}
$$

and while $t \in\left[T_{2}, T_{3}\right]$, in (4.1), $W^{*}(t)$ optimizes

$\int_{T_{2}}^{T_{3}} F\left(t, W, W^{\prime}\right) d t$ and is subject to

$$
W\left(T_{2}\right)=W_{T_{2}}, W\left(T_{3}\right)=W_{T_{3}}
$$

If there is no optimal solution in (4.3), (4.4) and (4.5), then we should know the subjection function is not a feasible solution. We will try another feasible solution in this horizon where $W^{*}(t)$ would change accordingly. Hence, if $W^{*}(t)$ is an optimal function in (4.1), it must also be optimal in (4.3), (4.4) and (4.5). Now, in the planning horizon $\left[T_{0}, T_{1}\right] 、\left[T_{1}, T_{2}\right] 、\left[T_{2}, T_{3}\right], W^{*}(t)$ can meet the Euler equation $F_{W}=d F_{W^{\prime}} / d t$. Although $W^{*}(t)$ meets the Euler equation in these three time zones, different endpoints would result in a different integral constant. So, if the value of either sub-problem could be improved, then the sum could be enhanced by a relevant segment of $W^{*}(t)$ via an improved solution of the subproblems. On the basis of the two endpoints $\left(T_{1}, W_{T_{1}}\right)$ and $\left(T_{2}, W_{T_{2}}\right)$, we can find an optimal solution $W^{*}(t)$ in (4.1) via the Corner Condition.

First, we consider the general condition. Suppose $T_{1}$, $T_{2}, W_{T_{1}}$ and $W_{T_{2}}$ can be selected to meet a target function in (4.2). Compared with $T_{1}, T_{2}, W_{T_{1}}$ and $W_{T_{2}}$, for any $\delta T_{1}$ 、 $\delta T_{2} 、 \delta W_{T_{1}} 、 \delta W_{T_{2}}$, if endpoint $\left(T_{1}, W_{T_{1}}\right)$ is (4.3) moved, then another endpoint $\left(T_{1}, W_{T_{1}}\right)$ in (4.4) will move accordingly because $W^{*}(t)$. Additionally, in (4.4), when the endpoint $\left(T_{2}, W_{T_{2}}\right)$ is moved, another endpoint $\left(T_{2}, W_{T_{2}}\right)$ in (4.5) is also moved accordingly. Prior to finding out the target function's variation in (4.2), $T_{1}$ 、 $T_{2} 、 W_{T_{1}}$ and $W_{T_{2}}$ changed. We need to find out its variation of sub-problems in (4.3)、 (4.4)、 and (4.5). From Kamien \& Schwartz (1991), we can understand this variation as the following model:

$$
J=\int_{T_{n-1}}^{T_{n}} F\left(t, W, W^{\prime}\right) d t
$$

In the target function, its variation $\delta J$ can be expressed as:

$\delta J=\left.\left(F-W^{\prime} F_{W^{\prime}}\right)\right|_{T_{n}} \delta T_{n}+\left.F_{W^{\prime}}\right|_{T_{n}} \delta W_{T_{n}}-\left.\left(F-W^{\prime} F_{W^{\prime}}\right)\right|_{T_{n-1}} \delta T_{n-1}-\left.F_{W^{\prime}}\right|_{T_{n-1}} \delta W_{T_{n-1}}$

$+\int_{T_{n-1}}^{T_{n}}\left(F_{W}-d F_{W^{\prime}} / d t\right) h d t$

Because all optimal solutions in these three subproblems need to meet the Euler equation, all integrals must be zero in (4.6). Furthermore, at time points $T_{0} 、 T_{1}$ 、 $T_{2}$ 、 and $T_{3}$, all are assumed to be fixed numbers, so, $\delta T_{0}=\delta T_{1}=\delta T_{2}=\delta T_{3}=0$. To simplify (4.6), the following can be written:

$$
\delta J=\left.F_{W^{\prime}}\right|_{T_{n}} \delta W_{T_{n}}-\left.F_{W^{\prime}}\right|_{T_{n-1}} \delta W_{T_{n-1}}
$$

In this paper, on the basis of management concepts, we summarize the optimal production function $W^{*}(t)$. Kuo-Chung Huang \& Chia-Pin Wu(2014):

To simplify the notation, we defined a 'decision function' as follows:

$$
\begin{gathered}
D F_{1}=D F_{1}\left(c_{1}, c_{2}, T_{1}, B_{1}\right)=B_{1}-\frac{c_{2} T_{1}^{2}}{4 c_{1}}, \\
D F_{2}=D F_{2}\left(c_{1}, c_{2}, T_{1}, T_{2}, B_{2}\right)=B_{2}-\frac{c_{2}\left(T_{2}-T_{1}\right)^{2}}{4 c_{1}}, \\
D F_{3}=D F_{3}\left(c_{1}, c_{2}, T_{2}, T_{3}, B_{3}\right)=B_{3}-\frac{c_{2}\left(T_{3}-T_{2}\right)^{2}}{4 c_{1}}
\end{gathered}
$$

\section{CONCLUSION}

With the advent of globalization, enterprises are increasingly challenged by complex internal operations. The traditional standardization involved in lane manufacturing is insufficient to save costs and satisfy customer needs. The best solution, therefore, lies in increasing capacity utilization while minimizing total costs. However, most workflow management systems are incapable of supporting dynamic programming, which cannot satisfy the increasing need for internal production management. In this paper, we designed and constructed a customized production system for an enterprise to design and schedule its own workflows within optimal control. Furthermore, by using the variation method and the method of transformation, we presented a set of functions and generated feasible solutions. Finally, the system was expertise in immediate or deferrable production scenarios to offer reference models for establishing dynamic production systems.

We point out the advantages and drawbacks for both the previous body of research and our own dynamic model described in this paper. On the basis of the structure of the model, we used dynamic control and calculus to obtain the optimal solution. This method provides a way for decision makers to attend to the best production starting point for any given time point while operating within the best production function. In this study, having acknowledged the limitations of the three- 
batch size, we can nevertheless confirm that it results in particular structural features.

\section{REFERENCES}

[1] Chen, M.S. \& Chu, M.C. (2003), The Analysis of Optimal Control in Matching Problem Between Manufacturing and Marketing, European Journal of Operational Research, Vol. 150, pp. 293-303.

[2] Chen, M.S. \& Tsai F.C. (2008), The Optimal Production Plan under Limited Production Capacity at Any Point in Time. Journal of the Operations Research Society of Japan, Vol.51, pp. 81-94.

[3] Chiang, A. (1992), Dynamic optimization, McGraw-Hill, Inc., Singapore.

[4] Flatto, L. (1979), Advanced Calculus, Second printing, Mei Ya Publications, Inc. Taipei, Taiwan.

[5] Friendman, A. (1983), Advanced Calculus, Fifth printing, Mei Ya Publications, Inc. Taipei, Taiwan.
[6] Gary M. K. \& Hector H.G..(1990), A conceptual model for demand management in the assemble-to-order environment, Journal of Operations Management, Vol 9, pp. 65-84.

[7] Grubbstrom, R.W. \& Wang, Z. (2003), A stochastic Model of Multi-Level/Multi-stage Capacity Constrained ProductionInventory Systems, International Journal of Production Economics, Vol. 81, pp. 483-494.

[8] Horiguchi, K., Raghavan, N., Uzsoy, R. \& Venkateswaran, S. (2001), Finite Capacity Production Planning Algorithms for a Semiconductor wafer fabrication facility, International Journal of Production Research, Vol.39, pp. 825-842.

[9] Kamien, M.I. \& Schwartz, N.L. (1991), Dynamic Optimization: The Calculus of Variations and Optimal Control in Economics and Management, Second Edition, Elsevier, North Holland.

[10] Kuo-Chung Huang \& Chia-Pin Wu, Dynamic Optimization Production Model in the Multi-stage of Make to Order Production Strategy, Advanced Materials Research Vol. 909 (2014) pp 293-304. 